\title{
Comparative evaluation of microfluidic circuit model performance for electroviscous flow
}

\author{
Christian J. C. Biscombe ${ }^{1} \quad$ Malcolm R. Davidson ${ }^{2}$ \\ Dalton J. E. Harvie ${ }^{3}$
}

(Received 30 January 2011; revised 14 July 2011)

\begin{abstract}
Microfluidic circuit models are useful tools for conceptualising and designing lab-on-chip devices. We evaluate the ability of two different microfluidic circuit models to accurately predict electroviscous (pressure driven) flow behaviour in a particular contraction-expansion geometry over an experimentally relevant range of inlet concentrations and surface charge densities. We show that a linear 'total current model' based on a relatively simple ion species constraint at circuit nodes performs well compared to a non-linear 'ion current model' that conserves species exactly. Specifically, the total current model overpredicts the total pressure and potential differences by less than $2 \%$ and $7 \%$ respectively for silica channels.
\end{abstract}

http://anziamj.austms.org.au/ojs/index.php/ANZIAMJ/article/view/3945 gives this article, (c) Austral. Mathematical Soc. 2011. Published July 26, 2011. ISSN 1446-8735. (Print two pages per sheet of paper.) Copies of this article must not be made otherwise available on the internet; instead link directly to this URL for this article. 


\section{Contents}

1 Introduction

C448

2 Problem specification

C451

3 Results and discussion

C453

4 Conclusions

C458

A Conductance coefficients

C459

References

C460

\section{Introduction}

The complexity of microfluidic devices (or labs-on-chip) has increased substantially in recent years, driven by an ever-broadening range of applications in chemistry and biotechnology $[1,6,13]$. With increasing device complexity comes an increasing demand for numerical tools, such as microfluidic circuit models, to aid design and optimisation [8]. Microfluidic circuit models are used to predict flow behaviour within a circuit by relating the driving forces (pressure and electric potential differences) to their conjugate fluxes (volume flow and current) in terms of known (or measurable) physicochemical properties [2].

Most solid surfaces acquire an electrostatic charge when brought into contact with an electrolyte solution [11]. This surface charge promotes a redistribution of ions within the liquid, resulting in the formation of a charged electric double layer (EDL) adjacent to the surface. If the ions are assumed to behave as point charges, then for a symmetric, binary, Newtonian electrolyte under isothermal conditions, the radial cation $(+)$ and anion $(-)$ concentration 
profiles obey Boltzmann distributions,

$$
n_{ \pm}=n_{0} \exp \left(\mp \frac{z_{ \pm} e}{k T} \psi(r)\right)
$$

Here, $z_{ \pm}$are the ion valencies, $e$ is the elementary charge, $k$ is the Boltzmann constant, $T$ is the (absolute) temperature, and $\psi(r)$ is the EDL potential. The factor $n_{0}=\sqrt{n_{+} n_{-}}$is the geometric mean ion concentration at any position within the channel $[9,12]$.

The equations describing fully developed, laminar, steady state, electrokinetic flow through a uniform microchannel are

$$
\left[\begin{array}{c}
\mathrm{Q} \\
\mathrm{I}
\end{array}\right]=\left[\begin{array}{ll}
\mathrm{L}_{11} & \mathrm{~L}_{12} \\
\mathrm{~L}_{21} & \mathrm{~L}_{22}
\end{array}\right]\left[\begin{array}{c}
\Delta \mathrm{p} \\
\Delta \phi
\end{array}\right],
$$

where $\mathrm{Q}$ is the volumetric flow rate, I is the total current, and $\Delta \mathrm{p}$ and $\Delta \phi$ are the axial pressure and potential differences along the channel, respectively [2]. All of the conductance coefficients $L_{i j}$ (where $1 \leqslant i, j \leqslant 2$ ) depend on the channel geometry and electrolyte properties (viscosity $\mu$, dielectric constant $\epsilon_{f}$, and ionic diffusivities $\mathrm{D}_{ \pm}$); all except $\mathrm{L}_{11}$ also depend on the temperature $\mathrm{T}$, surface charge density $\sigma_{d}$, and mean concentration $n_{0}$. Appendix A gives expressions for these coefficients.

For multiple channels connected in series (that is, end-to-end), conservation laws are applied at circuit nodes (channel junctions) to link together the fully developed flow solutions for each individual channel. Volume and total charge must be conserved across nodes, and a nodal constraint on the ion species is also required. We compare two different microfluidic circuit models, which we refer to as the 'total current model' (тсм) $[4,17]$ and the 'ion current model' (ICM) [5, 9], which are based on different nodal species constraints as outlined below. We presented a more rigorous treatment elsewhere [5].

Conservation of volume Both the TCM and ICM require that there be no accumulation of volume at nodes, that is

$$
\sum_{m=1}^{M} Q^{(m)}=0 \text {. }
$$


Here $M$ is the number of channels that intersect at the node, and $Q^{(m)}$ is the flow rate in branch $\mathrm{m}$ directed towards the node.

Conservation of total charge Similarly, both models conserve charge by requiring that the sum of total currents entering a node be zero

$$
\sum_{m=1}^{M} I^{(m)}=0
$$

Nodal constraint on ion species The two models impose different nodal species constraints.

- The TCM assumes that $\mathfrak{n}_{0}$ is uniform throughout the network and equal to the inlet concentration $n_{0}^{(\text {in) }}$ :

$$
\mathrm{n}_{0}^{(\mathrm{m})}=\mathrm{n}_{0}^{(\mathrm{in})} \text {. }
$$

This assumption is strictly valid only in a stationary system (that is, no net fluxes), but it is a fair approximation when the EDLs do not overlap in any of the channels [5]. The TCM is a linear model and is therefore comparatively straightforward to solve when the flow rate and total current are specified (as in the cases to be considered herein). Once $n_{0}^{\text {(in) }}$ is chosen, the conductance coefficients can be calculated immediately and the pressure and potential changes along each channel found from Equation (2) using (for example) Cramer's rule $[4,17]$.

- The ICM is instead based on the fact that each ionic species contributes a definite fraction of the total charge within the system. In order to conserve these ion charges, a stronger form of Equation (4) is adopted:

$$
\sum_{m=1}^{M} I_{ \pm}^{(m)}=0
$$


where $\mathrm{I}_{ \pm}$are ion currents due solely to the motion of one ionic species $\left(\mathrm{I}_{+}+\mathrm{I}_{-}=\mathrm{I}\right)$ [9]. The validity of Equation (6) is unaffected by the degree of EDL overlap. However, unlike the TCM, the value of $n_{0}$ in each channel is unknown a priori (that is, Equation (5) does not necessarily hold). Since $\mathrm{L}_{12}, \mathrm{~L}_{21}, \mathrm{~L}_{22}$ and $\mathrm{I}_{ \pm}$are all functions of $n_{0}$, the ICM is a non-linear model that cannot be solved via simple matrix manipulations.

In summary, the TCM (linear) comprises Equations (2)-(5), whilst the ICM (non-linear) comprises Equations (2), (3) and (6) (Equation (4) is implied by Equation (6)).

Due to the different nodal species constraints used by the TCM and ICM, the set of conductance coefficients $\mathrm{L}_{i j}$ for each of the two models may differ, which may in turn lead to differences between predictions for $\Delta p$ and $\Delta \phi$ (from Equation (2)). It is desirable to use the TCM whenever possible, because of its relative simplicity, but the range of concentrations and surface charge densities over which this model is accurate have not been reported previously. Therefore our aim is to determine the conditions under which the TCM performs acceptably for electroviscous (pressure driven) flow.

\section{Problem specification}

We consider a cylindrical contraction-expansion network (Figure 1) as an example of a simple series circuit. For electroviscous flow, the total current $\mathrm{I}=0$ at steady state. Model performance is evaluated by specifying the flow rate $\mathrm{Q}$ and inlet concentration $\mathrm{n}_{0}^{\text {(in) }}$ and then comparing predictions for the total pressure and potential differences, $\Delta \mathrm{p}_{\mathrm{t}}=\Delta \mathrm{p}^{\text {(in) }}+\Delta \mathrm{p}^{(\text {(on) }}+\Delta \mathrm{p}^{(\text {out })}$ and $\Delta \phi_{\mathrm{t}}=\Delta \phi^{\text {(in) }}+\Delta \phi^{\text {(con) }}+\Delta \phi^{\text {(out) }}$ respectively, occurring along the domain. Note that since the TCM and ICM are one dimensional models based upon the assumption of fully developed flow, entrance and exit effects near channel junctions are not included. 


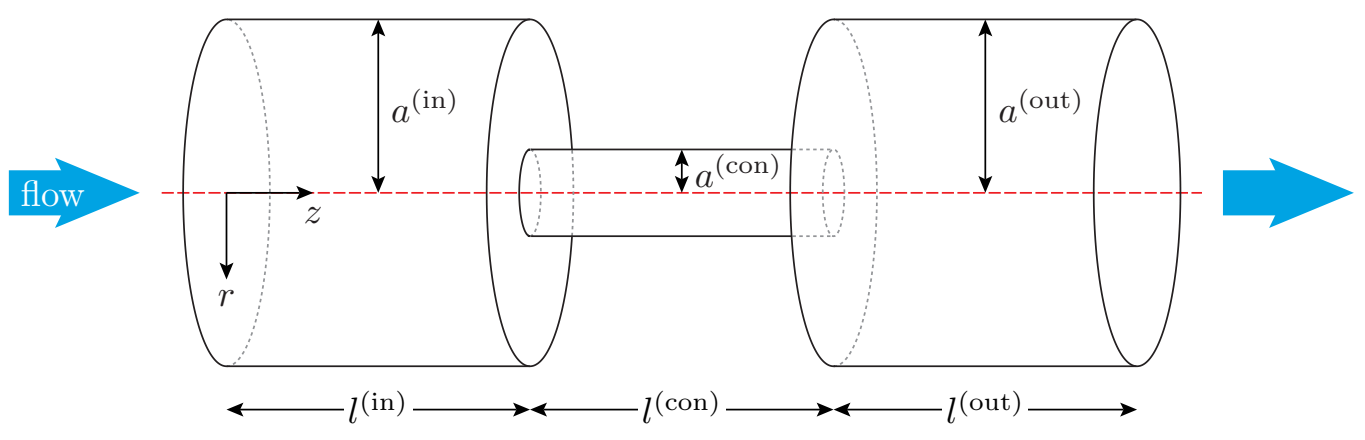

Figure 1: Schematic diagram of the contraction-expansion flow geometry (not to scale). Table 1 gives dimensions.

TABLE 1: Physical parameters and channel dimensions employed in this study. (Notes: $a$, data from Haynes [10]; $b$, based on properties of water.)

\begin{tabular}{lc}
\hline Property & Value \\
\hline $\mathrm{T}(\mathrm{K})$ & 298.15 \\
$\mu\left(10^{-4} \mathrm{~kg} \mathrm{~m}^{-1} \mathrm{~s}^{-1}\right)$ & $8.9002^{a, b}$ \\
$\epsilon_{\mathrm{f}}($ dimensionless $)$ & $78.408^{a, b}$ \\
$\mathrm{D}_{+}\left(10^{-9} \mathrm{~m}^{2} \mathrm{~s}^{-1}\right)$ & $1.957^{a}$ \\
$\mathrm{D}_{-}\left(10^{-9} \mathrm{~m}^{2} \mathrm{~s}^{-1}\right)$ & $2.032^{a}$ \\
\hline $\mathrm{a}^{\text {(in) }}=\mathrm{a}^{\text {(out) }}(\mu \mathrm{m})$ & 5.0 \\
$\mathrm{a}^{\text {(con })}(\mu \mathrm{m})$ & 0.050 \\
$\mathrm{l}^{\text {(in })}=l^{\text {(con })}=l^{\text {(out) }}(\mu \mathrm{m})$ & 5000.0 \\
\hline
\end{tabular}

The flow rate is fixed at $1 \mathrm{fl} \mathrm{s}^{-1}\left(10^{-18} \mathrm{~m}^{3} \mathrm{~s}^{-1}\right)$ (but our results are independent of the chosen flow rate, as we show in Section 3). Inlet concentrations ranging from $10^{-6} \mathrm{M}$ to $1 \mathrm{M}$ are considered, based on typical experimental studies $[14,15,16]$. All channels are assumed to possess a uniform surface charge density $\sigma_{\mathrm{d}}$, which we vary in magnitude from $10^{-6} \mathrm{C} \mathrm{m}^{-2}$ to $1 \mathrm{C} \mathrm{m}^{-2}$. Potassium chloride solution is selected as the electrolyte $\left(z_{+}=-z_{-}=1\right)$. Table 1 lists values of relevant physical parameters. 
The conductance coefficients for both models are calculated using a finite volume method [9]. A non-uniform mesh is used with 1,000 cells spanning the radius of each channel. We confirmed that our results are insensitive to further mesh refinement. For the ICM, $n_{0}^{(\text {con) }}$ is determined using an iterative bisection scheme.

\section{Results and discussion}

We use the term 'error' to mean the error in TCM predictions relative to ICM predictions, that is,

$$
\begin{aligned}
& \eta_{p}=\left|\frac{\Delta p_{t, T C M}-\Delta p_{t, I C M}}{\Delta p_{t, I C M}}\right|, \\
& \eta_{\phi}=\left|\frac{\Delta \phi_{t, T C M}-\Delta \phi_{t, I C M}}{\Delta \phi_{t, I C M}}\right|, \\
& \eta_{n_{0}}=\left|\frac{n_{0, \mathrm{TCM}}^{\text {(con })}-n_{0, I C M}^{(\text {con })}}{n_{0, \text { ICM }}^{\text {(con) }}}\right|,
\end{aligned}
$$

where $\mathrm{n}_{0, \mathrm{TCM}}^{(\mathrm{con})}=\mathrm{n}_{0}^{(\mathrm{in})}$ from Equation (5). For electroviscous flow, these errors are independent of the flow rate (provided that the flow remains laminar). For $\eta_{n_{0}}$ this independence occurs because $n_{0}$ is independent of $Q$, whilst for $\eta_{p}$ and $\eta_{\phi}$ this independence occurs because

$$
\begin{aligned}
& \Delta p^{(\mathfrak{m})}=\frac{\mathrm{L}_{22}^{(\mathfrak{m})}}{\lambda^{(\mathfrak{m})}} \mathrm{Q}, \\
& \Delta \phi^{(\mathfrak{m})}=-\frac{\mathrm{L}_{21}^{(\mathfrak{m})}}{\lambda^{(\mathfrak{m})}} \mathrm{Q},
\end{aligned}
$$

where $\lambda^{(\mathfrak{m})}=\mathrm{L}_{11}^{(\mathfrak{m})} \mathrm{L}_{22}^{(\mathfrak{m})}-\mathrm{L}_{12}^{(\mathfrak{m})} \mathrm{L}_{21}^{(\mathfrak{m})}$, so that $\mathrm{Q}$ cancels from both numerator and denominator in Equations (7) and (8). (Equations (10) and (11) are 
obtained by setting $\mathrm{I}=0$ in Equation (2) and rearranging terms.) The errors are also independent of the sign of the surface charge.

Error contours for TCM predictions of the total pressure and potential differences occurring along the contraction-expansion network are presented in Figures 2 and 3, respectively. Initially we focus on results specific to silica channels, taking $\sigma_{\mathrm{d}}=-25 \mathrm{mC} \mathrm{m}^{-2}$ as a typical value for silica [14] (indicated by the dashed lines in Figures 2 and 3). The peak errors for silica are $\eta_{p, \max }=0.0131$ and $\eta_{\phi, \max }=0.0602$, which in dimensional terms correspond to over-predictions of $19.7 \mathrm{kPa}$ and $60.6 \mathrm{mV}$ respectively (assuming all channels are $5 \mathrm{~mm}$ long). The peak errors occur at inlet concentrations of $6.5 \times 10^{-4} \mathrm{M}\left(\eta_{p, \max }\right)$ and $8.3 \times 10^{-4} \mathrm{M}\left(\eta_{\phi, \max }\right)$; both $\eta_{p}$ and $\eta_{\phi}$ decrease as the concentration is increased or decreased away from these points.

In reality, the surface charge of silica arises principally due to deprotonation of silanol groups, and as such $\sigma_{\mathrm{d}}$ for silica depends on the $\mathrm{pH}$ of the electrolyte solution $[3,16]$. Values of $\sigma_{\mathrm{d}}$ for silica previously quoted range from $-1 \mathrm{mC} \mathrm{m}^{-2}$ to $-192 \mathrm{mC} \mathrm{m}^{-2}[3,14,15,16]$. Within this range of surface charge densities, Figures 2 and 3 show that both $\eta_{p}$ and $\eta_{\phi}$ follow the same trends with concentration as described for the $-25 \mathrm{mC} \mathrm{m}^{-2}$ case, although the peak errors vary. At worst, $\eta_{p, \max }$ reaches 0.0250 , which is reasonably small, but $\eta_{\phi, \text { max }}$ reaches 0.167 , which may be unacceptable from a design perspective. We conclude that the TCM mostly yields acceptable results for $\Delta p_{\mathrm{t}}$ and $\Delta \phi_{\mathrm{t}}$ for electroviscous flow over the range of surface charge densities reported for silica, with the exception of predictions for $\Delta \phi_{\mathrm{t}}$ at intermediate concentrations and surface charge densities of order $-1 \mathrm{mC} \mathrm{m}^{-2}$.

Figures 2 and 3 show that for silica channels, the TCM and ICM agree closely (that is, $\eta_{p}, \eta_{\phi} \approx 0$ ) at the extreme ends of the concentration range. The agreement at high inlet concentrations is to be expected: when the EDLs do not overlap (as occurs at high concentrations), Equation (5) is a good approximation and the two models are essentially indistinguishable from one another. In other words, Equation (5) correctly conserves ions in this limit, as illustrated in Figure 4 by the convergence of $\eta_{n_{0}}$ to zero as $n_{0}^{\text {(in) }}$ becomes large. 


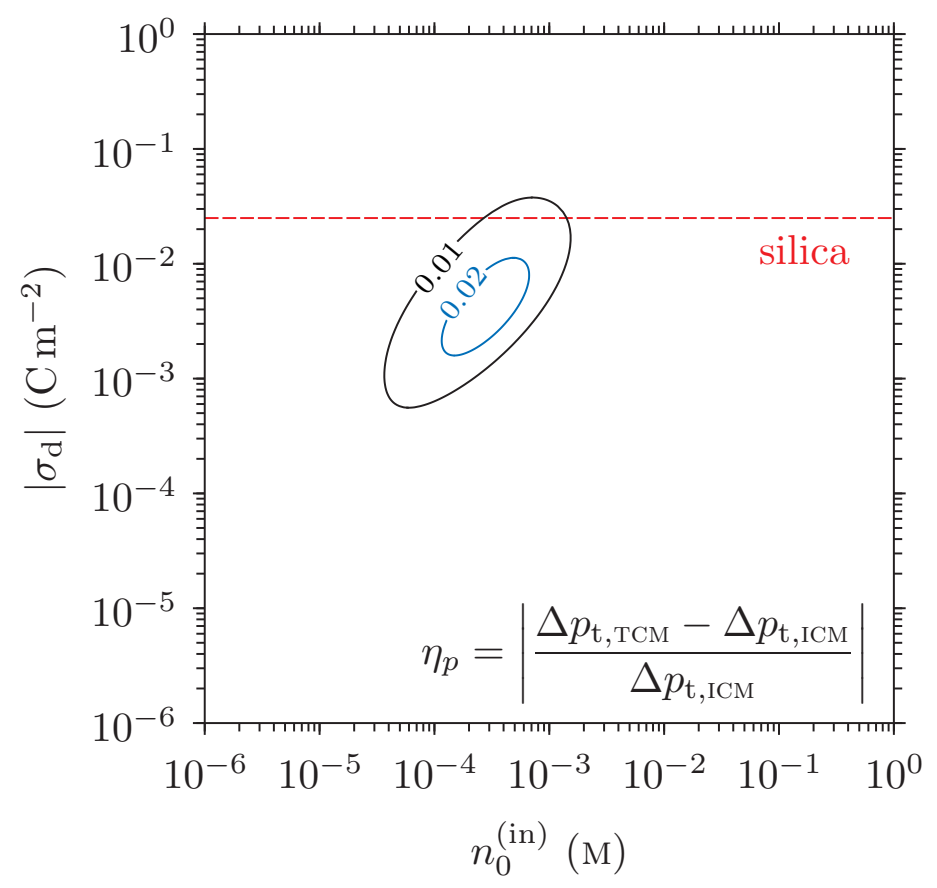

FIGURE 2: Error contours for TCM predictions of the total pressure difference along the circuit. The dashed line corresponds to $\sigma_{\mathrm{d}}=-25 \mathrm{mC} \mathrm{m}^{-2}$ (silica).

However, it is not immediately obvious why the two sets of predictions should also agree at low inlet concentrations. Given that the TCM is expected to be inaccurate when the EDLs overlap, one might expect the greatest discrepancies between the models to occur when the EDLs overlap most strongly, that is, at the lowest inlet concentrations. Indeed, Figure 4 shows that the values

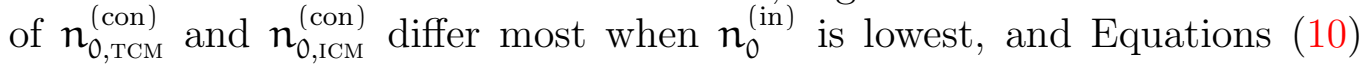
and (11) show that $\Delta \mathrm{p}^{(\mathrm{con})}$ and $\Delta \phi^{(\mathrm{con})}$ both depend on $\mathrm{n}_{0}^{\text {(con) }}$ (via $\mathrm{L}_{\mathfrak{i j}}^{(\mathrm{con})}$ ). This indicates that $\Delta \mathrm{p}^{\text {(con) }}$ and $\Delta \phi^{\text {(con) }}$ are only weakly dependent on $n_{0}^{\text {(con) }}$ (if at all) when $n_{0}^{\text {(in) }}$ is low.

Physically, we explain the weak $\mathrm{n}_{0}^{(\mathfrak{m})}$-dependences of $\Delta \mathrm{p}^{(\mathfrak{m})}$ and $\Delta \phi^{(\mathfrak{m})}$ in 


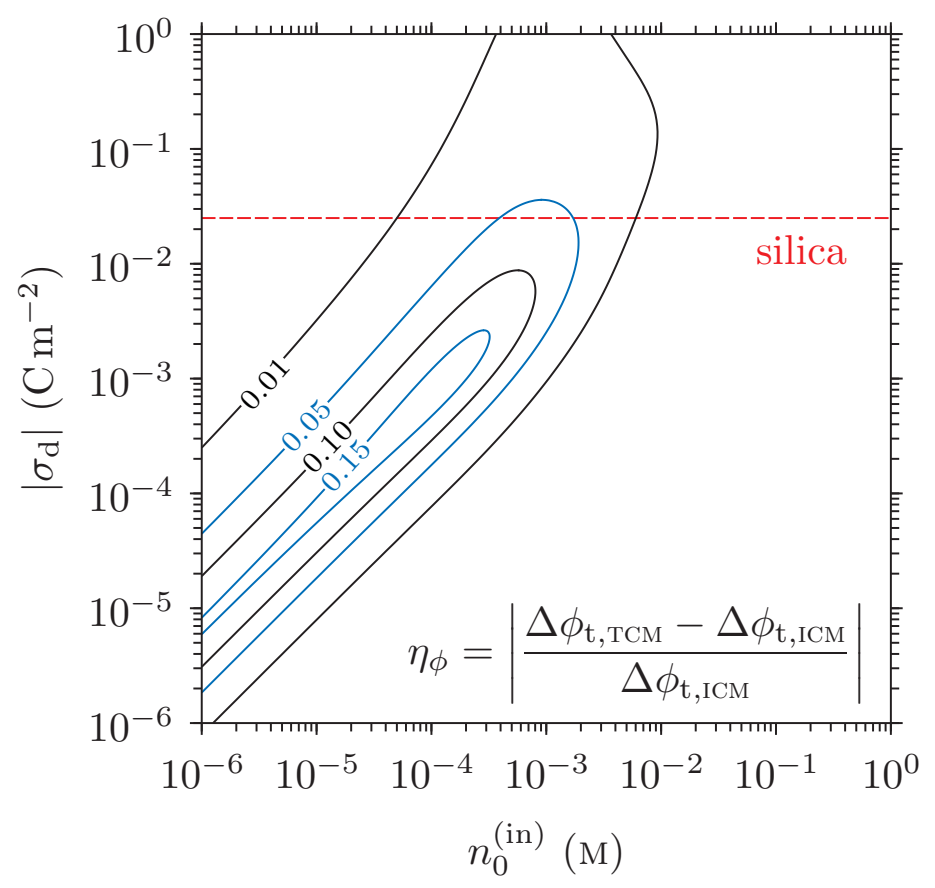

FIGURE 3: Error contours for TCM predictions of the total potential difference along the circuit. The dashed line corresponds to $\sigma_{\mathrm{d}}=-25 \mathrm{mC} \mathrm{m}^{-2}$ (silica).

silica channels at low inlet concentrations as follows. Equations (10) and (11) may be rewritten as

$$
\begin{gathered}
\Delta p^{(m)}=\frac{L_{22}^{(m)}}{L_{21}^{(m)}} \frac{I_{s}^{(m)}}{G_{e}^{(m)}} Q, \\
\Delta \phi^{(m)}=-\frac{I_{s}^{(m)}}{G_{e}^{(m)}} Q,
\end{gathered}
$$

where $\mathrm{I}_{s}^{(\mathfrak{m})}=\mathrm{L}_{21}^{(\mathfrak{m})} / \mathrm{L}_{11}^{(\mathfrak{m})}$ and $\mathrm{G}_{e}^{(\mathfrak{m})}=\lambda^{(\mathrm{m})} / \mathrm{L}_{11}^{(\mathfrak{m})}$. The terms $\mathrm{L}_{21}$ and $\mathrm{I}_{\mathrm{s}}$ represent streaming currents (since $\mathrm{L}_{21}=(\mathrm{I} / \Delta \mathrm{p})_{\Delta \phi=0}$ and $\mathrm{I}_{s}=(\mathrm{I} / \mathrm{Q})_{\Delta \phi=0}$ from Equation (2)), whilst $\mathrm{L}_{22}$ and $\mathrm{G}_{e}$ represent electrical conductances (since 


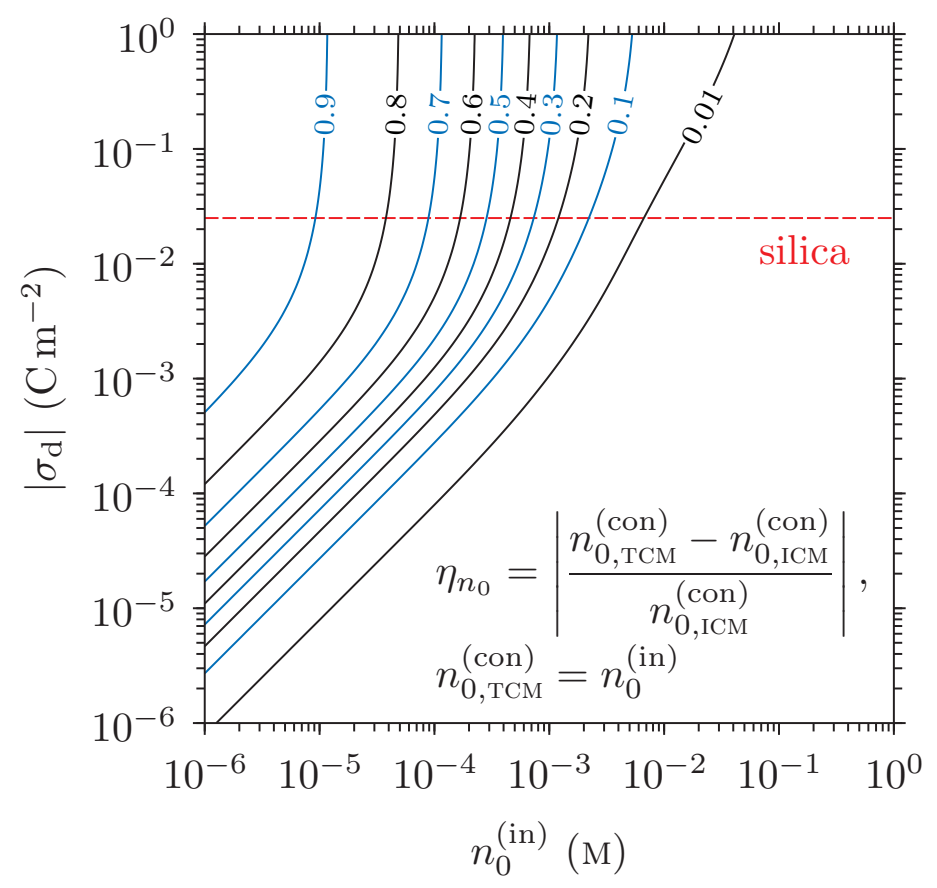

FIGURE 4: Error contours for TCM predictions of $n_{0}$ in the contraction. The dashed line corresponds to $\sigma_{\mathrm{d}}=-25 \mathrm{mC} \mathrm{m}^{-2}$ (silica).

$\mathrm{L}_{22}=(\mathrm{I} / \Delta \phi)_{\Delta \mathrm{p}=0}$ and $\mathrm{G}_{e}=(\mathrm{I} / \Delta \phi)_{\mathrm{Q}=0}$ from Equation (2)). These streaming currents and electrical conductances approach constant values in the dilute (low- $\left.n_{0}\right)$ limit $[7,14,15,16]$. The streaming current plateaux occur because the rate of charge advection (that is, the streaming current, by definition) is limited by the (constant) total charge present within the EDLs (of equal magnitude but opposite sign to the wall charge). The electrical conductance plateaux occur because a finite minimum number of counterions must be present in order to neutralise the surface charge. All quantities on the right-hand sides of Equations (12) and (13) therefore become constant as $\mathrm{n}_{0}^{(\mathfrak{m})}$ approaches zero, which implies that $\Delta \mathrm{p}^{(\mathrm{m})}$ and $\Delta \phi^{(\mathfrak{m})}$ tend towards constant values (independent of $n_{0}^{(m)}$ ) in the dilute limit. 
At very low surface charge densities $\left(\left|\sigma_{d}\right| \leqslant 10^{-4} \mathrm{C} \mathrm{m}^{-2}\right)$, Figure 3 shows that $\eta_{\phi}$ may be appreciable at low concentrations, in contrast to the behaviour for silica channels discussed above. This indicates that $\Delta \phi^{(\mathrm{m})}$ must depend on $n_{0}^{(m)}$ under these circumstances. As the surface charge is reduced, the streaming current and electrical conductance plateaux still occur, but the concentration at which the conductance begins to deviate from its plateau value decreases $[14,15]$. Thus, the large difference between $\boldsymbol{n}_{0, \text { TCM }}^{\text {(con) }}$ and $\boldsymbol{n}_{0, \text { ICM }}^{\text {(con) }}$ that occurs at low $n_{0}^{(i n)}$ (Figure 4 ) leads to significant differences between the predicted values of $\mathrm{G}_{e}^{(\mathrm{con})}$, and hence $\Delta \phi^{(\mathrm{con})}$, when the surface charge density is low. On the other hand, $\eta_{p}$ is essentially immune to this effect since $L_{12}^{(\text {con })}=$ $\mathrm{L}_{21}^{(\text {con })} \ll \mathrm{L}_{11}^{(\text {con })}, \mathrm{L}_{22}^{\text {(con) }}$ when $\sigma_{\mathrm{d}}$ is small (electrokinetic effects are insignificant at low surface charge densities), so that (using Equation (10)) $\Delta \mathrm{p}^{(\text {con) }}=$ $\mathrm{L}_{22}^{(\text {con })} \mathrm{Q} / \lambda^{(\text {con })} \approx \mathrm{Q} / \mathrm{L}_{11}^{(\text {con })}$, which is independent of $\mathrm{n}_{0}^{(\text {con })}$.

Finally, it is not possible to deduce the signs of the differences $\Delta \mathrm{p}_{\mathrm{t}, \mathrm{TCM}}-\Delta \mathrm{p}_{\mathrm{t}, \mathrm{ICM}}$, $\Delta \phi_{\mathrm{t}, \mathrm{TCM}}-\Delta \phi_{\mathrm{t}, \mathrm{ICM}}$, or $n_{0, \mathrm{TCM}}^{\text {(con) }}-n_{0, \mathrm{ICM}}^{\text {(con) }}$ that appear in Equations (7)-(9) by inspection of Figures 2-4 alone. However, we observed from our data that for the particular conditions considered herein, the TCM almost always overpredicts $\left|\Delta p_{t}\right|$ and $\left|\Delta \phi_{t}\right|$ and always under-predicts $n_{0}^{(\text {con })}$, relative to the ICM.

\section{Conclusions}

We evaluated the performance of two different microfluidic circuit models:

1. the TCM, which is based on a relatively simple nodal species constraint (Equation (5)) and which may be solved using straightforward linear algebra; and

2. the ICM (non-linear), which conserves species exactly (via Equation (6)) but which entails a more complex (iterative) numerical solution procedure. 
Compared to the ICM, the TCM over-predicts the total pressure and potential differences for electroviscous flow in a particular contraction-expansion network by less than 3\% and 17\% respectively over the full parameter space considered. The errors are largest at intermediate surface charge densities and low to moderate inlet concentrations. For the specific case of $\sigma_{\mathrm{d}}=-25 \mathrm{mC} \mathrm{m}^{-2}$, which is typical of silica channels, the TCM over-predicts the pressure and potential differences by less than $2 \%$ and $7 \%$ respectively, which are probably acceptable errors for design purposes. The TCM is less well suited to predicting the ion concentrations in the contraction; errors in $n_{0}^{(\operatorname{con})}$ may be large (>90\%), especially at high surface charge densities and low concentrations. This may limit the utility of the TCM for some applications. (The reported errors apply only for the specific channel sizes indicated in Table 1; altering the channel radii may alter the errors.)

Many microfluidic devices utilise electro-osmotic flow in conjunction with (or instead of) pressure driven flow [6]. We intend to examine how well the TCM performs for electrokinetic flow with non-zero total current in a future publication.

Acknowledgements This research was partly supported by an Australian Research Council Discovery Grant.

\section{A Conductance coefficients}

The conductance coefficients used in this study are defined as

$$
\begin{aligned}
& \mathrm{L}_{11}=-\frac{\pi \mathrm{a}^{4}}{8 \mu \mathrm{l}}, \\
& \mathrm{L}_{12}=\mathrm{L}_{21}=\frac{\pi \mathrm{a}^{4} z e n_{0}}{\mu \mathrm{l}} \mathcal{G}_{1},
\end{aligned}
$$




$$
\mathrm{L}_{22}=-\frac{\pi\left(\mathrm{a}^{2} \mathrm{zen}_{0}\right)^{2}}{\mu l}\left[\mathcal{G}_{3,+}+\mathcal{G}_{3,-}+\frac{\mu}{k \operatorname{Tn}_{0} \mathrm{a}^{2}}\left(\mathrm{D}_{+} \mathcal{G}_{4,+}+\mathrm{D}_{-} \mathcal{G}_{4,-}\right)\right] \text {, }
$$

where the non-dimensional $\mathcal{G}$ coefficients represent various integrals involving the radial ion concentration profiles [9]. The $\mathcal{G}$ coefficients are functions of $a$, $n_{0}, \mathrm{~T}, z, \epsilon_{\mathrm{f}}$ and $\sigma_{\mathrm{d}}$.

\section{References}

[1] P. Abgrall and A.-M. Gué. Lab-on-chip technologies: making a microfluidic network and coupling it into a complete microsystem - a review. J. Micromech. Microeng., 17:R15-R49, 2007. doi:10.1088/0960-1317/17/5/R01. C448

[2] A. Ajdari. Steady flows in networks of microfluidic channels: building on the analogy with electrical circuits. C. R. Phys., 5:539-546, 2004. doi:10.1016/j.crhy.2004.02.012. C448, C449

[3] S. H. Behrens and D. G. Grier. The charge of glass and silica surfaces. J. Chem. Phys., 115(14):6716-6721, 2001. doi:10.1063/1.1404988. C454

[4] C. L. A. Berli. Theoretical modelling of electrokinetic flow in microchannel networks. Colloids Surf., A, 301:271-280, 2007. doi:10.1016/j.colsurfa.2006.12.066. C449, C450

[5] C. J. C. Biscombe, M. R. Davidson, and D. J. E. Harvie. Microfluidic circuit analysis. II: Implications of ion conservation for microchannels connected in series, submitted to J. Colloid Interface Sci. C449, C450

[6] L. Bousse, C. Cohen, T. Nikiforov, A. Chow, A. R. Kopf-Sill, R. Dubrow, and J. W. Parce. Electrokinetically controlled microfluidic analysis systems. Annu. Rev. Biophys. Biomol. Struct., 29:155-181, 2000. doi:10.1146/annurev.biophys.29.1.155. C448, C459 
[7] H.-C. Chang and G. Yossifon. Understanding electrokinetics at the nanoscale: a perspective. Biomicrofluidics, 3(1):012001, 2009. doi:10.1063/1.3056045. C457

[8] D. Erickson. Towards numerical prototyping of labs-on-chip: modeling for integrated microfluidic devices. Microfluid. Nanofluid., 1:301-318, 2005. doi:10.1007/s10404-005-0041-z. C448

[9] D. J. E. Harvie, C. J. C. Biscombe, and M. R. Davidson. Microfluidic circuit analysis. I: Ion current relationships for thin slits and pipes, submitted to J. Colloid Interface Sci. C449, C451, C453, C460

[10] W. M. Haynes, editor. CRC Handbook of Chemistry and Physics (Internet version). CRC Press/Taylor and Francis, Boca Raton, Florida, USA, 91st edition, 2011. C452

[11] R. J. Hunter. Zeta Potential in Colloid Science: Principles and Applications. Academic Press, London, 1981. C448

[12] S. Levine, J. R. Marriott, and K. Robinson. Theory of electrokinetic flow in a narrow parallel-plate channel. J. Chem. Soc., Faraday Trans. 2, 71:1-11, 1975. doi:10.1039/F29757100001. C449

[13] K. Ohno, K. Tachikawa, and A. Manz. Microfluidics: applications for analytical purposes in chemistry and biochemistry. Electrophoresis, 29:4443-4453, 2008. doi:10.1002/elps.200800121. C448

[14] R. B. Schoch and P. Renaud. Ion transport through nanoslits dominated by the effective surface charge. Appl. Phys. Lett., 86(25):253111, 2005. doi:10.1063/1.1954899. C452, C454, C457, C458

[15] D. Stein, M. Kruithof, and C. Dekker. Surface-charge-governed ion transport in nanofluidic channels. Phys. Rev. Lett., 93(3):035901, 2004. doi:10.1103/PhysRevLett.93.035901. C452, C454, C457, C458 
[16] F. H. J. van der Heyden, D. Stein, and C. Dekker. Streaming currents in a single nanofluidic channel. Phys. Rev. Lett., 95(11):116104, 2005. doi:10.1103/PhysRevLett.95.116104. C452, C454, C457

[17] X. Xuan and D. Li. Analysis of electrokinetic flow in microfluidic networks. J. Micromech. Microeng., 14:290-298, 2004. doi:10.1088/0960-1317/14/2/018. C449, C450

\section{Author addresses}

1. Christian J. C. Biscombe, Department of Chemical and Biomolecular Engineering, The University of Melbourne, Parkville, Victoria 3010, Australia.

2. Malcolm R. Davidson, Department of Chemical and Biomolecular Engineering, The University of Melbourne, Parkville, Victoria 3010, Australia.

3. Dalton J. E. Harvie, Department of Chemical and Biomolecular Engineering, The University of Melbourne, Parkville, Victoria 3010, Australia.

mailto:daltonh@unimelb.edu.au 